\title{
Ergodic Properties of Plane Billiards with Symmetric Potentials
}

\author{
Roberto Markarian \\ Instituto de Matemática y Estadística, "Prof. Ing. Rafael Laguardia," Facultad de Ingeniería, \\ CC No. 30, Montevideo, Uruguay
}

Received September 10, 1990

\begin{abstract}
We study chaotic behaviour of the motion of a particle moving like in a billiard table outside some disks where a symmetric potential acts. Quadratic forms introduced in (Markarian, 1988) to study non-vanishing Lyapunov exponents are used.
\end{abstract}

\section{Introduction}

A. The purpose of this paper is to prove some ergodic properties (see C. below) of dynamical systems defined by the motion of a point mass in a bounded connected region $Q$ of the plane. The particle moves like in a billiard table outside some disk contained in $Q$, and under the action of symmetric potentials inside them.

B. This problem was discussed (on the 2-torus) in Sinai (1963). In Kubo (1976) and Kubo and Murata (1981) it is proved that some conditions on the rotation function (see Sect. 2 of this paper) are sufficient for the ergodicity (and the Bernoulli property) of the dynamical system on the torus; Kubo mentions some repelling potentials that verify these conditions.

During the International Conference and Workshop on Dynamical Systems, I.M.P.A., Rio de Janeiro, August 1989, Victor Donnay announced some results (obtained by himself and Carlangelo Liverani) on the same subject, using invariant cone techniques; and Yacob Sinai suggested me to study related problems using quadratic forms, a method I used to deduce conditions on the boundaries for chaotic billiards.

C. Let $\mu$ be a probability measure on a compact 2-manifold $M, K$ a subset such that $\mu(K)=0, H=M \backslash K$ and $T: H \rightarrow H$ the restriction to $H$ of a $\mathbb{C}^{r}$-diffeomorphism, $r \geq 1$, defined on an open subset of $M$ that preserved the measure $\mu$. We will assume that

$$
\log ^{+}\left\|\left(T^{ \pm 1}\right)_{x}^{\prime}\right\| \in L^{1}(H, \mu) \quad\left(\log ^{+} s=\max \{\log s, 0\}\right)
$$

a condition that permits to apply the ergodic multiplicative theorem of Oseledets.

$\Sigma(T)$ will denote the Pesin region, that is the set of regular points that have only non-zero Lyapunov exponents. 
If $\mu(\Sigma(T))=1$ we will say that the map $T$ (or the dynamical system it defines is non-uniform hyperbolic or has chaotic behaviour. In Markarian (1990) a characterization of non-uniform hyperbolicity of smooth maps with singularities in terms of Lyapunov quadratic forms is given.

$B: T M \rightarrow \mathbb{R}$ is a quadratic form on $M$ if $B_{x}: T_{x} M \rightarrow \mathbb{R}$ is a quadratic form in the usual sense. If $f: M \rightarrow M$ is a diffeomorphism we denote by $f^{\#} B$ (pullback of $B$ by $f$ ) the quadratic form $\left(f^{\#} B\right)_{x} u=B_{f(x)}\left(f_{x}^{\prime} u\right) . B$ is non-degenerate on a subset $N \subset M$ if $B_{x}$ is non-degenerate for every $x \in N$, that is, the matrix associate to $B_{x}$ with respect to any base and the inner product has non-zero eigenvalues. $B$ is positive in $N$ if $B_{x} u>0$ for every $x \in N$ and every non-zero $u \in T_{x} M$.

Theorem. Let $B: T M \rightarrow R$ be a quadratic form such that $B_{x}$ depends measurably on $x$, is non-degenerate in $H$ and $P_{x}=\left(T^{\#} B-B\right)_{x}$ is positive for every $x \in H$. Then $\mu(\Sigma(T))=1$.

A slightly different version of the theorem $\left(B_{x}\right.$ depends continuously on $x$ on a set of measure one) was proved in Markarian (1988).

D. It is well known that the existence of non-zero Lyapunov exponents for $T$ allows to construct locally invariant submanifolds. If all Lyapunov exponents are different from zero in a set $\Lambda$, a non-uniform hyperbolic decomposition of $T_{x} M$ is obtained at each point $x \in \Lambda$ and if $\Lambda$ has positive measure there is a countable number of invariant sets $\Lambda_{i}$ of positive measure $\left(\Sigma \mu\left(\Lambda_{i}\right)=\mu(\Lambda)\right)$ such that $\left.T\right|_{\Lambda_{i}}$ is ergodic. See, for instance Pesin and Sinai (1981) for a survey of such results. The ergodicity of $T$ follows from the uniqueness of the ergodic components $\Lambda_{i}$. And this can be done studying the behaviour of $T$ on $K$, the set of its singularities.

So, applying the theorem in order to have non-vanishing Lyapunov exponents on sets of measure one we will construct quadratic forms. Conditions on the elements that determine the dynamical system will appear as a consequence of the hypothesis on the quadratic forms.

E. To study the dynamical system that is the object of this paper we will work on the space $M_{1}$ of the pairs $(q, v)=x$, where $q$ is a point of the set $\mathscr{B}$ union of the regular components of the boundary of $Q$ and the circumferences determined by the disks where the potentials are defined, and $v$ is the velocity vector of the trajectory leaving $q . T x$ will be the pair (if it exists) defined by the position and the velocity of the particle at the next "leaving position" on $\mathscr{B}$.

Then we study the action of $T^{\prime}$ on the tangent space to $M_{1}$. This is done on a special parametrization of $T M_{1}$ (inspired on the study of geodesic flows) that allows to write the quadratic forms in a very simple way.

Conditions on the rotation function and on the geometry of the problem will appear after establishing the positivity of

$$
P=T^{\#} B-B \text {. }
$$

This is studied in general in Sect. 3 and in a particular example $(U(r)=U$ (const)) in Sect. 4, where some conjectures proposed in Baldwin (1988) are proved.

We do not study in general the potentials admissible with those rotation functions. However we indicate the relationship between rotation functions (and their derivatives) and $U(r)$ in some particular important cases.

In order to have ergodic properties of the flow determined by the motion, some conditions that must be imposed on the potential are evident: there cannot be sets of positive measure of trajectories that finish or are contained in the disks. 


\section{Central Potentials in a Circle}

Let $Q$ be a bounded, connected region of the plane whose boundary consists of a finite set of curves $\partial Q_{i}, \mathbb{C}^{r+1}, r \geq 2$ with curvature $\left(\left|k_{i}\right|\right)$ bounded. The regular components of the boundary $\partial \tilde{Q}_{i}=\partial Q_{i} \backslash \bigcup_{i \neq j} \partial Q_{j}$ have positive (focusing components), negative (dispersing) or zero curvature (neutral).

Let $\mathscr{D}$ be a disk completely contained in $Q$, with boundary $\mathscr{C}$ such that $d\left(\mathscr{C}, \partial Q_{i}\right)$ $>0$ for every $i, d$ is the euclidean distance in $\mathbb{R}^{2}$. In $\mathscr{D}$ is defined a central potential $U(r)$.

We consider the motion of a point unit mass that is reflected elastically in $\partial \tilde{Q}=$ $\bigcup \partial \tilde{Q}_{i}$ which moves freely with velocity of norm one outside the disk, and under the action of the potential inside the disk.

If $n(q)$ is the unit inward normal (relative to $Q \backslash \mathscr{D}$ ) in $q \in \partial \tilde{Q} \cup \mathscr{C}=\mathscr{B}$, then the parametrization $q(s)$ and the curvature $K(s)$, where $s$ is the arc length on $\mathscr{B}$, are defined by the equations

$$
q^{\prime \prime}(s)=K \vec{n}=K i q^{\prime}(s),
$$

where $i$ is the $\pi / 2$-angle counter clockwise rotation operator, $K=-\frac{1}{R}$ on $\mathscr{C}$.

In order to determine the movement inside the circle we consider its equations with energy $E=\frac{1}{2}$, and angular momentum $M$. Its position in polar coordinates $(r(t), \varphi(t))$ is determined by

$$
M=r^{2} \dot{\varphi}, \quad \frac{1}{2}=\frac{1}{2}\left(\dot{r}^{2}+r^{2} \dot{\varphi}^{2}\right)+U(r),
$$

with initial conditions $(r(0), \varphi(0))=\left(R, \varphi_{2}\right)=\bar{q}_{2}$ (Fig. 1). See, for example, Landau and Lifchitz (1981, Sect. 14).

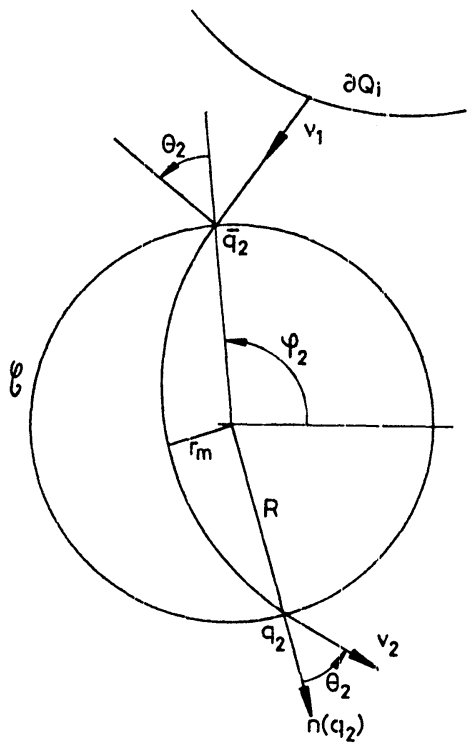



have

If $M \neq 0$ there is a closest distance of approach to the center $r_{m}=r(T)$ and we

$$
\dot{r}=\sqrt{1-2 U(r)-M^{2} / r^{2}}, \quad r \in\left[r_{m}, R\right]
$$

and, finally

$$
\frac{d \varphi}{d r}=\frac{M}{r \sqrt{(1-2 U(r)) r^{2}-M^{2}}} .
$$

If $\theta_{2}$ is the angle of "reflection" - not properly the angle of incidence (see Fig. 1) - the angular momentum evaluated at $t=0$ and

$$
\left.t=T \quad \text { is } \quad M=\mid\left(\bar{q}_{2}-0\right)\right) \wedge \vec{v}_{1} \mid=R \sin \theta_{2}=\sqrt{r_{m}^{2}\left(1-2 U\left(r_{m}\right)\right)},
$$

supposing $\theta_{2} \in[0, \pi / 2)$.

The symmetry of the potential implies that a particle that enters at the point $\bar{q}_{2}$ with the indicated angle $\theta_{2}$ will leave the disk at $q_{2}=\left(R, \varphi_{2}+g\left(\theta_{2}\right)\right)$. The angle between $n\left(q_{2}\right)$ and the velocity $v_{2}$ of the particle is $\theta_{2}$.

In the following section we will take coordinates $(s, \theta)$ on the phase space. In this case $s$ is measured clockwise on the circumference; so if we take the same origin ( $\varphi=0$, for example) it results $s=-R \varphi$.

The function $g:\left(-\frac{\pi}{2}, \frac{\pi}{2}\right) \rightarrow \mathbb{R}$ is called the rotation function. It depends on the potentials $U(r)$ in the following way:

$$
g\left(\theta_{2}\right)=2 \int_{r_{m}}^{R} \frac{M d r}{r \sqrt{(1-2 U(r)) r^{2}-M^{2}}} \quad \text { if } \quad \theta_{2} \in(0, \pi / 2) .
$$

Since a particle that enters in the disk with angle $\theta_{2} \in(0, \pi / 2)$ will rotate counter clockwise the same amount that an orbit entering with angle $-\theta_{2}$ will rotate clockwise, for $-\frac{\pi}{2}<\theta<0$ we define $g(\theta)=-g(-\theta)$. Thus, in general, $g$ is discontinuous at $\theta=0$ and we will ignore the trajectories that enter the disk with angle $\theta_{2}=0$; they determine a set of measure zero on our phase space. If $\lim _{\theta_{2} \rightarrow 0^{+}} g\left(\theta_{2}\right)=n \pi$, for any
$n \in \mathbb{Z}$ we are able to define a continuous function:

$$
g\left(\theta_{2}\right)=2 n \pi-g\left(-\theta_{2}\right) \quad \text { if } \quad-\frac{\pi}{2}<\theta_{2} \leq 0
$$

We suppose that $G\left(\theta_{2}\right)=g^{\prime}\left(\theta_{2}\right)$ exists for every $\theta_{2} \neq 0$.

Since it can be useful for applications, we calculate explicitly $G\left(\theta_{2}\right)$ in the case $U(r)$ continuous for $r \in(0, R], U(R)=0, U^{\prime}(r)$ continuous for $r \in(0, R)$. Some additional conditions must be imposed in order to have convergent integrals.

We study first the relation between $\theta_{2}$ and $r_{m}$. Studying the velocity of the particle at $q_{2}, t=2 T$, we obtain

$$
\cos \theta_{2}=\frac{\dot{r}(2 T)}{\sqrt{R^{2} \dot{\varphi}^{2}(2 T)+\dot{r}^{2}(2 T)}} .
$$

From the energy equation it results $1=\dot{r}^{2}(2 T)+R^{2} \dot{\varphi}^{2}(2 T)$. So $\dot{r}^{2}(2 T)=1-$ $M^{2} / R^{2}$ and $\cos \theta=\frac{\sqrt{R^{2}-M^{2}}}{R}$. 
Supposing $\theta_{2}>0$, we have $M, \dot{\varphi}>0$ and $\theta_{2}=\operatorname{Arcos} \frac{\sqrt{R^{2}-M^{2}}}{R}$ with $M=$ $r_{m}\left(1-2 U\left(r_{m}\right)\right)^{1 / 2}$. So

$$
\frac{d \theta_{2}}{d r_{m}}=\frac{r_{m}\left(1-2 U\left(r_{m}\right)-r_{m} U^{\prime}\left(r_{m}\right)\right)}{M \sqrt{R^{2}-M^{2}}}
$$

In order to calculate $\frac{d g}{d r_{m}}$ is better to make the substitution $r=e^{s} r_{m}$. Then

$$
g\left(r_{m}\right)=2 \int_{0}^{\log R / r_{m}} \frac{\left(1-2 U\left(r_{m}\right)\right)^{1 / 2} d s}{\left[e^{2 s}\left[1-2 U\left(e^{s} r_{m}\right)\right] e^{2 s}-1+2 U\left(r_{m}\right)\right]^{1 / 2}}
$$

and

$$
\frac{d g}{d r_{m}}=\frac{-2\left(1-2 U\left(r_{m}\right)\right)^{1 / 2}}{\sqrt{R^{2}-M^{2}}}+\frac{2 r_{m}}{M} \int_{0}^{\log R / r_{m}} I(s) d s
$$

with

$$
I(s)=\frac{e^{3 s}\left(1-2 U\left(r_{m}\right)\right) U^{\prime}\left(e^{s} r_{m}\right)-e^{2 s}\left(1-2 U\left(e^{s} r_{m}\right)\right) U^{\prime}\left(r_{m}\right)}{\left[e^{2 s}\left[1-2 U\left(e^{s} r_{m}\right)\right]-1+2 U\left(r_{m}\right)\right]^{3 / 2}} .
$$

And finally

$$
G\left(\theta_{2}\right)=\frac{-2\left(1-2 U\left(r_{m}\right)\right)}{1-2 U\left(r_{m}\right)-r_{m} U^{\prime}\left(r_{m}\right)}+\frac{2 \sqrt{R^{2}-M^{2}}}{1-2 U\left(r_{m}\right)-r_{m} U^{\prime}\left(r_{m}\right)} \int_{0}^{\log R / r_{m}} I(s) d s .
$$

\section{Dynamics and Chaotic Behaviour}

As usually, let be

$$
M_{1}=\{(q, v): q \in \mathscr{B},\|v\|=1,\langle v, n(q)\rangle>0\} .
$$

Given $x_{1}=\left(q_{1}, v_{1}\right) \in M_{1}, T x_{1}$ (if defined) is defined moving forward in the direction $v_{1}$, a distance (time) $t_{1}$ till the intersection either with $\partial \tilde{Q}$ in $q_{2}$ or with $\mathscr{C}$ in $\bar{q}_{2}$. In the first case let $v_{2}$ be the reflected vector: $v_{2}=v_{1}-2\left\langle n\left(q_{2}\right), v_{1}\right\rangle n\left(q_{2}\right)$; in the second one, let $q_{2}$ be as in the previous section, and $v_{2}=e^{i \theta_{2}} n\left(q_{2}\right)$, where $e^{i \theta_{2}}$ is the rotation operator of angle $\theta_{2}\left(e^{i \pi / 2}=i\right)$. In any case $T x_{1}=x_{2}=\left(q_{2}, v_{2}\right)$. We do not define it on $S$, determined by the $x_{i} \in M$, such that $q_{2} \in \partial Q \backslash \partial \tilde{Q}$, or $v_{1}$ is tangent to $\mathscr{C}$ or $\theta_{2}=0$ if $\bar{q}_{2} \in \mathscr{C}$. Let $K=T^{k} S, k \in \mathbb{Z}$. If as usual $d \mu=d \theta d s \cos \theta$, normalized and $H=M_{1} \backslash K$ it results that $T: H \rightarrow H$ is measurable, $\mathbb{C}^{r}$ diffeomorphism and $\mu(K)=0$.

Small modifications must be done in the proof of Lemma 1, Chap. 6 of Cornfeld etal. (1982) in order to prove that $T$ is $\mu$-measure preserving. The proof that $T$ verifies the general conditions on the map $f$ of Sect. 1 and that it is a discontinuous dynamical system (or a smooth map with singularities) are given in details in Part V (Plane billiards as smooth dynamical systems, by J.M. Strelcyn) of Katok and Strelcyn (1986).

A wave front is given by the curve $(q(s), v(s))$ in $M_{1}$ and so an element in the tangent space at $x=(q(0), v(0)) \in M_{1}$ is $\left(q^{\prime}(0), v^{\prime}(0)\right)=\left(q^{\prime}, v^{\prime}\right)$. We restrict now the 
analysis to the case $q_{1} \in \partial \tilde{Q}, q_{2} \in \mathscr{C}$. The other two cases $\left(q_{1}, q_{2} \in \partial \tilde{Q}, q_{1} \in \mathscr{C}\right.$, $\left.q_{2} \in \partial \tilde{Q}\right)$ are studied as the billiard map.

Let be $\gamma_{1}, \gamma_{2}$ the parametrization of $\partial \tilde{Q}$ and $\mathscr{C}$, respectively, with parameters the arc lengths; $s_{1}, s_{2}$ the arc lengths of $\gamma_{1}, \gamma_{2}$ in neighbourhoods of $q_{1}, \bar{q}_{2} ; \theta_{j}\left(s_{j}\right)$ the angles of $n\left(q_{j}\left(s_{j}\right)\right)$ with $v_{j}\left(s_{j}\right)$; and $K_{j}\left(s_{j}\right)$ the curvatures in $q_{j}\left(s_{j}\right), j=1,2$. In this analysis $K_{2}=-\frac{1}{R}$.

If $q^{\prime}$ and $v^{\prime}$ are projected on $i v$, the positive $\pi / 2$-rotated of $v$, we obtain a natural parametrization of the tangent space. Let be $V_{j}=\left\langle v_{j}^{\prime}, i v_{j}\right\rangle, \alpha_{j}=\left\langle q_{j}^{\prime}, i v_{j}\right\rangle, j=1,2$. We have that

$$
\begin{gathered}
q_{1}\left(s_{1}\right)=\gamma_{1}\left(s_{1}\right), \quad s_{2}=s_{2}\left(s_{1}\right), \\
v_{1}\left(s_{1}\right)=i \gamma_{1}^{\prime}\left(s_{1}\right) e^{i \theta_{1}\left(s_{1}\right)}=-i \gamma_{2}^{\prime}\left(s_{2}\right) e^{-\theta_{2}\left(s_{2}\right)}, \\
\bar{q}_{2}\left(s_{2}\right)=\gamma_{1}\left(s_{1}\right)+t_{1}\left(s_{1}\right) v_{1}\left(s_{1}\right), \\
q_{2}\left(s_{2}\right)=\gamma_{2}\left(s_{2}-R g\left(\theta_{2}\left(s_{2}\right)\right)=0+e^{i g\left(\theta_{2}\left(s_{2}\right)\right)}\left(\gamma_{2}\left(s_{2}\right)-0\right),\right. \\
v_{2}\left(s_{2}\right)=i \gamma_{2}^{\prime}\left(s_{2}-\operatorname{Rg}\left(\theta_{2}\left(s_{2}\right)\right) e^{i \theta_{2}\left(s_{2}\right)} .\right.
\end{gathered}
$$

If we denote $\frac{d s_{2}}{d s_{1}}=\sigma^{\prime}, \frac{d \theta_{2}}{d s_{2}}=\theta_{2}^{\prime}, \frac{d \theta_{1}}{d s_{1}}=\theta_{1}^{\prime}$ and take derivatives with respect to $s_{1}$ in the previous formulas we obtain from (1),

$$
i \gamma_{1}^{\prime \prime}\left(s_{1}\right) e^{i \theta_{1}\left(s_{1}\right)}-\gamma_{1}^{\prime}\left(s_{1}\right) \theta_{1}^{\prime}\left(s_{1}\right) e^{i \theta_{1}\left(s_{1}\right)}=\sigma^{\prime}\left[-i \gamma_{2}^{\prime \prime}\left(s_{2}\right)-\gamma_{2}^{\prime}\left(s_{2}\right) \theta_{2}^{\prime}\left(s_{2}\right)\right] e^{-i \theta_{2}\left(s_{2}\right)},
$$

and so

$$
K_{1}+\theta_{1}^{\prime}=\sigma^{\prime}\left[K_{2}-\theta_{2}^{\prime}\right]
$$

and from (2), taking products by $-i v_{1}=-\gamma_{2}^{\prime} e^{-i \theta_{2}}$,

$$
\begin{gathered}
\left\langle\gamma_{2}^{\prime} \sigma^{\prime},-\gamma_{2}^{\prime} e^{-i \theta_{2}}\right\rangle=\left\langle\gamma_{1}^{\prime}+t_{1}^{\prime} v_{1}+t_{1} v_{1}^{\prime}, \gamma_{1}^{\prime} e^{i \theta_{1}}\right\rangle \\
-\sigma^{\prime} \cos \theta_{2}=\cos \theta_{1}-t_{1}\left(K_{1}+\theta_{1}^{\prime}\right)
\end{gathered}
$$

and so

$$
\cos \theta_{1}=\sigma^{\prime}\left[t_{1}\left(K_{2}-\theta_{2}^{\prime}\right)-\cos \theta_{2}\right]
$$

Finally,

$$
\begin{aligned}
\alpha_{2}= & \left.\left\langle q_{2}^{\prime}, i v_{2}\right\rangle=\left\langle\gamma_{2}^{\prime}\left(s_{2}-R g\left(\theta_{2}\right)\right)\left(1-R g^{\prime}\left(\theta_{2}\right) \theta_{2}^{\prime}\right) \sigma^{\prime}, i \gamma_{2}^{\prime}\left(s_{2}-R g\left(\theta_{2}\right)\right) e^{i \theta_{2}}\right)\right\rangle \\
= & -\cos \theta_{2}\left(1-R g^{\prime}\left(\theta_{2}\right) \theta_{1}^{\prime}\right) \sigma^{\prime} \\
V_{2}= & \left\langle v_{2}^{\prime}, i v_{2}\right\rangle=\left\langle i \gamma_{2}^{\prime \prime}\left(s_{2}-R g\left(\theta_{2}\right)\right)\left(1-R g^{\prime}\left(\theta_{2}\right) \theta_{2}^{\prime}\right) \sigma^{\prime} e^{i \theta_{2}}\right. \\
& \left.-\gamma_{2}^{\prime}\left(s_{2}-R g\left(\theta_{2}\right)\right) \theta_{2}^{\prime} \sigma^{\prime} e^{i \theta_{2}},-\gamma_{2}^{\prime}\left(s_{2}-R g\left(\theta_{2}\right)\right) e^{i \theta_{2}}\right\rangle \\
= & \sigma^{\prime}\left[K_{2}\left(1-R g^{\prime}\left(\theta_{2}\right) \theta_{2}^{\prime}\right)+\theta_{2}^{\prime}\right] \\
V_{1}= & \left\langle v_{1}^{\prime}, i v_{1}\right\rangle=K_{1}+\theta_{1}^{\prime}=\sigma^{\prime}\left(K_{2}-\theta_{2}^{\prime}\right) \\
\alpha_{1}= & \left\langle q_{1}^{\prime}, i v_{1}\right\rangle=-\cos \theta_{1}=\sigma^{\prime}\left[\cos \theta_{2}-t_{1}\left(K_{2}-\theta_{2}^{\prime}\right)\right] .
\end{aligned}
$$

The general expression of a quadratic form $B_{x}, x=(q, v)$ is $B_{x}\left(q^{\prime}, v^{\prime}\right)=a \alpha^{2}+$ $2 b \alpha V+c V^{2}$, where $a, b, c$ are functions of $s, \theta$ (using the natural parametrization of $M_{1}$ ). In order to verify condition (ii) of Theorem $1, \mathrm{a}, \mathrm{b}$ and $\mathrm{c}$ must be measurable 
functions of $x$ and $a c-b^{2} \neq 0$. We take $b=1$ in order to have control of nondegeneracy of $B$ and to simplify the calculus. We will use $\theta$ instead of $\theta_{2}, K$ instead of $K_{2}$ and $G$ instead of $g^{\prime}\left(\theta_{2}\right)$. Then

$$
\begin{aligned}
P_{x_{1}}\left(q^{\prime}, v^{\prime}\right) & \\
= & \left(T^{\#} B-B\right)_{x_{1}}\left(q^{\prime}, v^{\prime}\right)=a_{2} \alpha_{2}^{2}-2 \alpha_{2} V_{2}+c_{2} V_{2}^{2}-a_{i} \alpha_{1}^{2}-2 \alpha_{1} V_{1}-c_{1} V_{1}^{2} \\
= & \left(a_{2}-a_{1}\right) \alpha_{2}^{2}+\left(c_{2}-c_{1}\right) V_{2}^{2}+a_{1}\left(\alpha_{2}^{2}-\alpha_{1}^{2}\right)+2\left(\alpha_{2} V_{2}-\alpha_{1} V_{1}\right)+c_{1}\left(V_{2}^{2}-V_{1}^{2}\right) \\
= & \sigma^{\prime 2}\left\{\theta ^ { \prime 2 } \left[\left(a_{2}-a_{1}\right) R^{2} G^{2} \cos ^{2} \theta+\left(c_{2}-c_{1}\right)(1-K R G)^{2}+a_{1}\left(R^{2} G^{2} \cos ^{2} \theta-t_{1}^{2}\right)\right.\right. \\
& \left.-2 K R^{2} G^{2} \cos \theta+2 R G \cos \theta+2 t_{1}+c_{1}\left(K^{2} R^{2} G^{2}-2 K R G\right)\right] \\
& +2 \theta^{\prime}\left[-\left(a_{2}-a_{1}\right) R G \cos ^{2} \theta+\left(c_{2}-c_{1}\right)\left(K-K^{2} R G\right)\right. \\
& \left.+a_{1}\left(K t_{1}^{2}-R G \cos ^{2} \theta-t_{1} \cos \theta\right)+2 K R G \cos \theta-2 K t_{1}+c_{1}\left(2 K-K^{2} R G\right)\right] \\
& +\left(a_{2}-a_{1}\right) \cos ^{2} \theta+\left(c_{2}-c_{1}\right) K^{2}+a_{1}\left(2 K t_{1} \cos \theta-K^{2} t_{1}^{2}\right) \\
& \left.-4 K \cos \theta+2 K^{2} t_{1}\right\} . \\
\text { If } a_{i}=0, P>0 \text { if and only if } & \quad\left[\left(c_{2}-c_{1}\right)(1-K R G)^{2}-2 K R^{2} G^{2} \cos \theta+2 R G \cos \theta\right. \\
& \left.\quad+2 t_{1}+c_{1}\left(K^{2} R^{2} G^{2}-2 K R G\right)\right] \theta^{\prime 2}+2 \theta^{\prime}\left[\left(c_{2}-c_{1}\right)\left(K-K^{2} R G\right)\right. \\
& \left.\quad+2 K R G \cos \theta-2 K t_{1}+c_{1}\left(2 K-K^{2} R G\right)\right] \\
& \quad+\left(c_{2}-c_{1}\right) K^{2}-4 K \cos \theta+2 K^{2} t_{1}>0,
\end{aligned}
$$

and this is true if and only if

$$
\left(c_{2}-c_{1}\right) K^{2}-4 K \cos \theta+2 K^{2} t_{1}>0,
$$

and,

$$
\begin{aligned}
& {\left[c_{2}\left(K-K^{2} R G\right)+c_{1} K+2 K R G \cos \theta-2 K t_{1}\right]^{2}} \\
& \quad-\left[c_{2}(1-K R G)^{2}-c_{1}-2 K R^{2} G^{2} \cos \theta+2 R G \cos \theta+2 t_{1}\right] \\
& \quad \times\left[\left(c_{2}-c_{1}\right) K^{2}-4 K \cos \theta+2 K^{2} t_{1}\right]<0 .
\end{aligned}
$$

If $c_{i}=0$, these inequalities become $(K=-1 / R)$,

$$
\begin{gathered}
\frac{2 \cos \theta}{R}+\frac{t_{1}}{R^{2}}>0 . \\
\left(-2 G \cos \theta+2 \frac{t_{1}}{R}\right)^{2}-\left(2 R G^{2} \cos \theta+2 R G \cos \theta+2 t_{1}\right)\left(\frac{4 \cos \theta}{R}+\frac{2 t_{1}}{R^{2}}\right)<0 .
\end{gathered}
$$

The first one is always true and the second one is

$$
\cos \theta\left\{-G(G+2) \cos \theta-\frac{t_{1}}{R}(G+1)(G+2)\right\}<0 .
$$

As $\cos \theta>0$ it must be $(G+2)\left[R G \cos \theta+t_{1}(G+1)\right]>0$ which is true either if $G<-2$ or $G>\frac{-t_{1}}{t_{1}+R \cos \theta}>-1$.

If $G=-2$, very simple considerations allow to deduce that each trajectory goes out the disk as it does not exist; that is $v_{1}=v_{2}$. If the case $Q$ were a square, we have a dynamical system that has vanishing Lyapunov exponents and is not ergodic. 
Indeed, if $G=-2$, the expression that must be studied to know the sign of $P$ is $\left(a_{i}=c_{i}=0\right)$,

$$
\begin{aligned}
& \left(4 R \cos \theta+2 t_{1}\right) \theta_{1}^{2}+2\left(4 \cos \theta+\frac{2 t_{1}}{R}\right)+\frac{4}{R} \cos \theta+\frac{2 t_{1}}{R} \\
& =\left(\frac{4}{R} \cos \theta+\frac{2}{R^{2}} t_{1}\right)\left(R \theta^{\prime}+1\right)^{2}>0 \quad \text { if } \quad \theta^{\prime} \neq-\frac{1}{R}=K .
\end{aligned}
$$

From (5), $\theta^{\prime}=\theta_{2}^{\prime}=K_{2}$ implies $\theta_{1}^{\prime}=-K_{1}=0$. Now consider the wave front that has entered the disk with $\theta_{2}^{\prime}=K_{2}$ : at the moment it leaves the circle it has $\bar{\theta}_{1}=-K_{2}$ because the circulation on the circumference of inward and outward trajectories are opposite. Then, as (5) is valid for billiards, at the next bounce against the rectilinear wall, we have $\theta_{2}^{\prime}=0$. This implies that $P=0$ along these trajectories and we cannot apply Theorem 1 .

We study now what happens if $-2<G<\frac{-t_{1}}{t_{1}+R \cos \theta}$. By looking at expression (8) we deduced that the positivity of $P$ is not difficult to study when $c_{i}=c$ (const). In this case $P$ is positive if and only if

$$
\frac{(G+2)}{R^{2}}\left\{4 R \cos \theta\left[-R G \cos \theta-t_{1}(G+1)\right]+c(G+2)\left(c-2 t_{1}\right)\right\}<0 .
$$

As we are supposing $-R G \cos \theta-t_{1}(G+1)>0$, the expression between the parenthesis is smaller than

$$
-4 R\left[R G+t_{1}(G+1)\right]+c(G+2)\left(c-2 t_{1}\right) .
$$

We suppose now, for example, that $t_{1} \geq c$; then a sufficient condition for verifying (9) is

$$
-4 R[R G-c(G+1)]-c^{2}(G+2)<0 ;
$$

and, if $G+2 \geq d>0, f(c)=d c^{2}+4 R c(d-1)+4 R^{2}(d-2)>0$.

But $f(c)=d(c+2 R)\left[c-\frac{2 R(2-d)}{d}\right]$ which is positive, for positive $c$, if

$$
c>\frac{2 R(2-d)}{d}=c_{1}
$$

This is not a good condition since $c_{1} \rightarrow+\infty$ if $d \rightarrow 0$ but the method used indicates how to work in specific problems. (9) is

As another example, observe that if $t_{1}>\frac{c}{2}$, a sufficient condition for verifying

$$
-R G-\frac{c}{2}(G+1)<0 ;
$$

and if $G>-1$, this is verified if

$$
c>\frac{-2 R G}{G+1} .
$$

If $q_{2} \in \partial \tilde{Q}_{i}$, the positivity of $P$ must be studied as in the billiard map. This was done in our paper (Markarian 1990), formulas (6), (7), and $\left(7^{\prime}\right)$, where it is proved 
that sufficient and necessary condition for $P$ being positive are

$$
\begin{gathered}
c_{2}-c_{1}-a_{1} t_{1}^{2}+2 t_{1}>0, \\
\left(c_{1}-2 t_{1}+a_{1} t_{1}^{2}\right)\left(4 c_{2}-2 E+\left(a_{2}-a_{1}\right) \frac{E^{2}}{4}\right) \\
+c_{2} E\left(2-2 a_{1} t_{1}-\left(a_{2}-a_{1}\right) \frac{E}{4}\right) \\
+\frac{a_{1}^{2} t_{1}^{2} E^{2}}{4}<0, \quad \text { with } E=\frac{2 \cos \theta}{K_{2}} ; \quad \text { if } \quad K_{2} \neq 0, \\
\left(a_{2}-a_{1}\right)\left(c_{1}-c_{2}-2 t_{1}\right)+a_{1} a_{2} t_{1}^{2}<0, \quad \text { if } \quad K=0 .
\end{gathered}
$$

We have used two quadratic forms:

$$
\begin{array}{ll}
a_{i}=0, & c_{i}=0 \\
a_{i}=0, & c_{i}=c \text { (const). }
\end{array}
$$

In both cases formula (10) is verified, $\left(12^{\prime}\right)$ is transformed in an equality and $P=0$ for a value of $\theta^{\prime}$.

But if almost all trajectories enter in the disk - and thus $P>0$ - there is no problem with this "bad" wave front. This is the case when $Q$ is the unit square centered in the center of $\mathscr{C}$ with $R<1 / 2$.

If $K_{2}<0$, (12) is verified and we can use any type of $C^{3}$ dispersing curve as $\partial Q_{i}$, obtaining non-uniform hyperbolic systems.

We can also combine neutral and dispersing components as parts of $\mathscr{B} \backslash \mathscr{C}$ if we take care of not generating sets of positive measure of trajectories that bounce only at the neutral ones.

\section{Constant Potentials}

In this section we study some of the results conjectured in Baldwin, 1988) for constant potentials $U(r)=U$ (const), $0 \leq r<R$ in the torus. See Sect. 5 of his paper and remember that only chaotic behaviour is observed by numerical methods; not ergodicity. As he works with total energy $E=1$, his $U$ corresponds to our $2 U$.

We calculate first the value of $G\left(\theta_{2}\right)=g^{\prime}\left(\theta_{2}\right)$.

Inside the disk the trajectory is rectilinear. For $\theta_{2} \in[0, \pi / 2$ ), we have (Fig. 2, observe how we measure $\bar{\theta}_{2}$ ) $r_{m}=R \sin \bar{\theta}_{2}$ and the angular momentum is

$$
M=R \sin \theta_{2}=R \sin \bar{\theta}_{2} \sqrt{1-2 U} .
$$

So

$$
g\left(\theta_{2}\right)=2 \int_{r_{m}}^{R} \frac{M d r}{r \sqrt{(1-2 U) r^{2}-M^{2}}}
$$

and, if

$$
\begin{gathered}
z^{2}=\frac{1-2 U}{M^{2}} r^{2}-1, \\
g\left(\theta_{2}\right)=2 \int_{r_{M}}^{R_{M}} \frac{d z}{z^{2}+1}=2 \operatorname{Arctg} \sqrt{\frac{1-2 U}{\sin ^{2} \theta_{2}}-1}=2 \operatorname{Arcos} \frac{\sin \theta_{2}}{\sqrt{1-2 U}}
\end{gathered}
$$




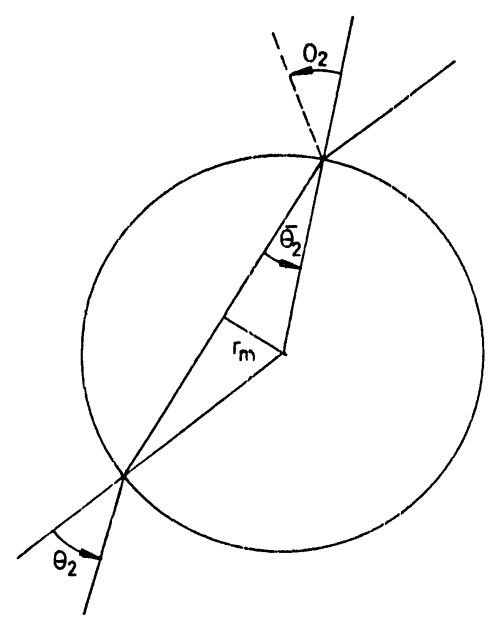

Fig. 2

(we take the determination of Arcos $z$ such that $\pi \geq \operatorname{Arcos} z \geq 0$ ). In order to make $g$ continuous at $\theta_{2}=0$, we take, for $\theta_{2} \in(-\pi / 2,0), g\left(\theta_{2}\right)=2 \pi-g\left(-\theta_{2}\right)$.

Observe that if $U>0$, for certain angles $\left(\sin \theta_{2}>\sqrt{1-2 U}\right)$ the expression of $g\left(\theta_{2}\right)$ does not make sense. In these cases the particle does not enter in the disk: it is reflected as in a billiard, and the graph of $g$ is as in Fig. 3

Fig. 3

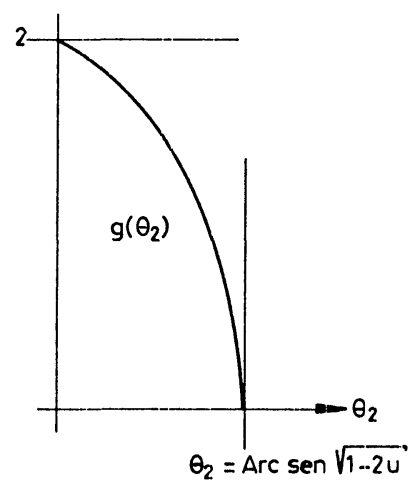

If $g\left(\theta_{2}\right)$ is defined,

$$
G\left(\theta_{2}\right)=\frac{-2 \cos \theta_{2}}{\sqrt{\cos ^{2} \theta_{2}-2 U}}
$$

which graph is in Fig. 4a for $U<0$, and in Fig. 4b for $0<U<1 / 2$.

Observe that from the formula for $V_{1}, \alpha_{1}$, that are valid for billiards, as we calculate $P$, neutral components can be ignored if $t_{1}$ indicates time between two successive touchs with $\mathscr{C}$. Indeed, if $K_{1}=0$ and the subindex 0 is used for all the elements in 
the previous (to $q_{1}$ ) bounce, then

$$
\begin{gathered}
K_{0}+\theta_{0}^{\prime}=\frac{d s_{1}}{d s_{0}}\left(K_{1}-\theta_{1}^{\prime}\right)=-\frac{d s_{1}}{d s_{0}} \theta_{1}^{\prime}=-\frac{d s_{2}}{d s_{0}}\left(K_{2}-\theta_{2}^{\prime}\right) ; \\
-\cos \theta_{0}=\frac{d s_{1}}{d s_{0}}\left[\cos \theta_{1}-t_{0}\left(K_{1}-\theta_{1}^{\prime}\right)\right] \\
=\frac{d s_{2}}{d s_{0}}\left[-\cos \theta_{2}+\left(t_{0}+t_{1}\right)\left(K_{2}-\theta_{2}^{\prime}\right)\right] .
\end{gathered}
$$

So, the study of chaotic behaviour of a particle moving on a torus, freely outside a disk and submitted to the action of a potential inside, it can be considered as in a square $Q$. If the square has unit sides, and the disk has radius $R$, both centered in $(0,0)$, then $t_{1} \geq 2\left(\frac{1}{2}-R\right)=1-2 R$.

If $U(r)=U$, we must distinguish two cases:

a) $0<U<1 / 2$. In this case we use the quadratic form with $a_{1}=c_{1}=0$. If the particle enters in the disk, $G<-2$ (see Fig. 4b) and $P>0$. If it does not enter, it is reflected as in a billiard, and from our previous work (Markarian 1988, Sect. 2.A), it is deduced again that $P>0$. So in this case the dynamical system has chaotic behaviour.

Fig. 4
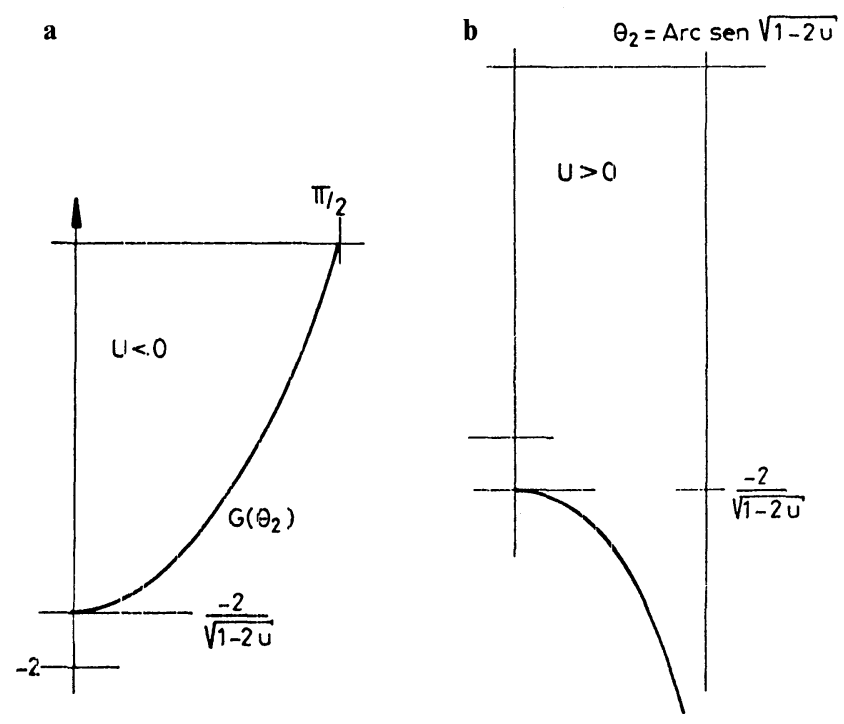

b) $U<0$. We use the quadratic form with $a_{i}=0, c_{1}=c$ (constant).

As $G \geq \frac{-2}{\sqrt{1-2 U}}$, we take in (10) $d=\frac{-2+2 \sqrt{1-2 U}}{\sqrt{1-2 U}}$, and so $c_{1}=$ $\frac{2 R}{-1+\sqrt{1-2 U}}$. As $t_{1} \geq 1-2 R$, we can take $c=1-2 R$ if $1-2 R>\frac{2 R}{-1+\sqrt{1-2 U}}$.

This implies $2 U<1-\left(\frac{1}{1-2 R}\right)^{2}=U_{l}$. 
Fig. 5

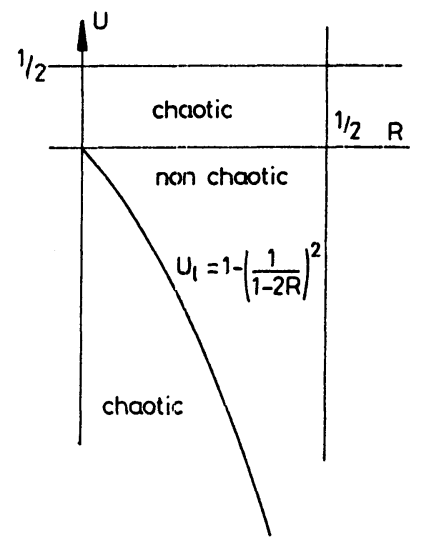

Baldwin proved - using KAM theory - that the system neither is ergodic nor has Pesin region of measure one if $2 U>U_{l}$. He conjectured (5.4) that the system is ergodic for $2 U<u_{l}$; we have proved that it has chaotic behaviour (see Fig. 5).

In this case the quadratic form $B\left(q^{\prime}, v^{\prime}\right)=2 \alpha V+(1-2 R) V^{2}$ is the best one since it allows to deduce chaotic behaviour in the maximal set $2 U<U_{l}$.

As I was revising this paper I knew that A. Knauf had proved the results in Sect. 4 using invariant cones techniques: Physica D 36, 259-262 (1989).

Acknowledgements. This work was begun at IMPA, Rio de Janeiro, in December 1989. I maintained useful conversations with Victor Donnay, Jorge Lewowicz, and Yacob Sinai.

\section{References}

Baldwin, P.R.: Soft billiard systems. Physica D 29, 321-342 (1988)

Cornfeld, I.P., Fomin, S.V., Sinai, Ya.G.: Ergodic theory. Berlin, Heidelberg, New York: Springer 1982

Donnay, V., Liverani, C.: Potentials on the Turo-Torus for which the Hamiltonian flow is ergodic. Commun. Math. Phys. 135, 267-302 (1991)

Katok, A., Strelcyn, J.-M. In: collaboration with Ledrappier, F., Przytyky, F.: Smooth maps with singularities. Invariant manifolds, entropy and billiards. Lect. Notes in Math. vol. 1222. Berlin, Heidelberg, New York: Springer 1986

Kubo, I.: Perturbed billiard systems. I. The ergodicity of the motion of a particle in a compound central field. Nagoya Math. J. 61, 1-57 (1976)

Kubo, I., Murata, H.: Perturbed billiard systems. II. Bernoulli properties. Nagoya Math. J. 81, 1-25 (1981)

Landau, L., Lifchitz, E.: Mécanique. Moscou: MIR 1966

Markarian, R.: Billiards with Pesin region of measure one. Commun. Math. Phys. 118, 87-97 (1988)

Markarian R.: Non-uniform hyperbolic billiards. Tese de doutoramento. IMPA, Rio de Janeiro 1990

Pesin, Ya.B., Sinai, Ya.G.: Hyperbolicity and stochasticity of dynamical systems. Soviet scientific reviews, Sect. C. Math. Phys. Rev. 4, 53-115 (1981)

Sinai, Ya.G.: On the foundations of the ergodic hypothesis for a dynamical system of statistical mechanics. Sov. Math. Dokl. 4, 1818-1822 (1963)

Sinai, Ya.G.: Dynamical systems with elastic reflections. Russ. Math. Surv. 25, 137-189 (1970)

Wojtkowski, M.: Principles for the design of billiards with nonvanishing Lyapunov exponents. Commun. Math. Phys. 105, 391-414 (1986) 